\title{
Composição Florística do Estrato Arbóreo na RPPN Porangaba em Itaguaí, Estado do Rio de Janeiro, Brasil
}

\author{
Mateus Figueira Gandra, André Felippe Nunes-Freitas, Michel de Souza Schütte \\ Laboratório de Ecologia Florestal e Biologia Vegetal, Departamento de Ciências Ambientais, \\ Instituto de Florestas, Universidade Federal Rural do Rio de Janeiro - UFRRJ
}

\begin{abstract}
RESUMO
Foi realizado o levantamento florístico do estrato arbóreo na RPPN Porangaba (Itaguaí, RJ). Foram amostradas 50 parcelas de $100 \mathrm{~m}^{2}$, nas quais todos os indivíduos com DAP $\geq 5 \mathrm{~cm}$ foram mensurados. Foram amostrados 943 indivíduos (877 vivos), distribuídos em 36 famílias, 66 gêneros e 105 espécies. As espécies com maior VI foram: Guarea guidonia (L.) Sleumer, Piptadenia gonoacantha (Mart.) J.F. Macbr, Casearia sylvestris Sw. e Bathysa gymnocarpa K. Schum. As famílias mais representativas foram Fabaceae $(S=14)$, Myrtaceae $(S=11)$ e Moraceae $(S=6)$. A área guarda uma representativa diversidade da floresta submontana, mas ainda são necessários estudos sobre a dinâmica da comunidade vegetal da RPPN Porangaba.
\end{abstract}

Palavras-chave: fitossociologia, mata secundária, Mata Atlântica, RPPN Porangaba, Itaguaí.

\section{Floristic Composition of Tree Layer at RPPN Porangaba in Itaguaí, State of Rio de Janeiro, Brazil}

\begin{abstract}
We have performed a floristic inventory of the tree layer at RPPN Porangaba (Itaguaí, RJ). We have sampled 50 plots of $100 \mathrm{~m}^{2}$ where all individual trees with $\mathrm{DBH} \geq 5 \mathrm{~cm}$ were measured. We have sampled 943 individual trees ( 877 births), distributed in 36 families, 66 genera and 105 species. The species with higher VI were Guarea guidonia (L.) Sleumer, Piptadenia gonoacantha (Mart.) J.F. Macbr, Casearia sylvestris Sw. and Bathysa gymnocarpa K. Schum. The most representative families were Fabaceae $(S=14)$, Myrtaceae $(S=11)$ and Moraceae $(S=6)$. The area holds a representative diversity of submontane forest, but studies are needed on the dynamics of plant community of RPPN Porangaba.
\end{abstract}

Keywords: phytossociology, secondary forest, Atlantic forest, RPPN Porangaba, Itaguaí.

\section{INTRODUÇÃO}

A Floresta Atlântica, em sua distribuição geográfica original, estendia-se ao longo da costa brasileira em uma área de aproximadamente 1.300.000 $\mathrm{km}^{2}$, desde o Rio Grande do Norte até o Rio Grande do Sul, numa faixa de largura variada, abrangendo uma pequena porção dos territórios paraguaio e argentino (Morellato \& Haddad, 2000), estando presente tanto nas planícies costeiras, como nas encostas e planaltos (Morellato \& Haddad, 2000; Peixoto et al., 2002). No entanto, durante 
cinco séculos, a Floresta Atlântica vem sofrendo os impactos negativos de vários ciclos econômicos (Dean, 1997; Morellato \& Haddad, 2000; Rocha et al., 2003) e, mais recentemente, da expansão urbana e da especulação imobiliária.

Estimativas sugerem que a área florestada do Estado do Rio de Janeiro era de $4.294 \mathrm{~km}^{2}$, o equivalente a $97 \%$ da área do Estado, que, após sofrer uma redução contínua de sua cobertura florestal, encontra-se bastante fragmentada, com apenas 20\% do seu tamanho original (Fundação SOS Mata Atlântica/INPE, 2006). No entanto, devido a sua elevada diversidade biológica, os remanescentes florestais do Rio de Janeiro podem ser considerados como um hotspot dentro do hotspot Floresta Atlântica (Rocha et al., 2003), destacando-se como região de enorme importância para a conservação (Morellato \& Haddad, 2000).

Apesar da reduzida área coberta por florestas, o Rio de Janeiro ainda abriga porções exuberantes da Mata Atlântica, que, além de extraordinária biodiversidade, concentram monumentos e sítios naturais únicos na sua paisagem, beleza e relevância cultural (Rocha et al., 2003), sendo considerado pela UNESCO como Reserva da Biosfera (Rambaldi et al., 2003). Os remanescentes florestais do Estado do Rio de Janeiro podem ser divididos em cinco grandes blocos, sendo o bloco Sul Fluminense um dos que guarda uma das maiores áreas florestadas, abrangendo os municípios de Paraty, Angra dos Reis, Mangaratiba, Rio Claro, parte de Itaguaí e a porção leste do Rio de Janeiro (Rocha et al., 2003). Este bloco guarda uma das maiores áreas de floresta contínua conservada do Estado e diversas Unidades de Conservação Federais e Estaduais (Rocha et al., 2003).

O município de Itaguaí sofreu entre os anos de 1994 e 2001, uma redução de $42 \%$ da sua cobertura vegetal, sendo considerada uma das áreas que sofreu maior taxa de perda de vegetação no Estado do Rio de Janeiro (TCE-RJ, 2004). No entanto, pouco ainda se conhece sobre os remanescentes florestais desse município, o que dificulta as ações de conservação.

Neste cenário, a Reserva Particular do Patrimônio Natural Porangaba figura como uma área estratégica para a conservação da diversidade biológica do município de Itaguaí. Localizada na
Serra do Caçador, esta RPPN guarda uma porção importante de um remanescente de vegetação submontana, que liga outras manchas de vegetação tão importantes quanto esta área. Este estudo teve por objetivo realizar o levantamento florístico do estrato arbóreo da Reserva Particular do Patrimônio Natural Porangaba (RPPN Porangaba), onde se encontra um importante remanescente de vegetação submontana do município de Itaguaí. Foram analisadas a composição, riqueza, diversidade e a estrutura da vegetação, de forma a identificar o estado de conservação do fragmento florestal onde está inserida esta RPPN. Para isso, objetivamos responder às seguintes perguntas: i) Qual a composição, riqueza e diversidade do componente arbóreo da RPPN Porangaba? ii) Qual a estrutura da comunidade arbórea da RPPN Porangaba? iii) Qual o grau de similaridade do componente arbóreo da RPPN Porangaba em relação a outras áreas localizadas na região e outras localidades?

\section{MATERIAL E MÉTODOS}

\section{2. Área de estudo}

O estudo foi realizado de fevereiro de 2007 a janeiro de 2008 na Reserva Particular do Patrimônio Natural Porangaba (22 $48^{\prime} 11^{\prime \prime} \mathrm{S}$ e $43^{\circ} 49^{\prime} 42^{\prime}$ O), localizada no município de Itaguaí, Região Sul Fluminense, na extremidade mais setentrional da Costa Verde. A RPPN Porangaba representa a associação de três RPPNs adjacentes, denominadas RPPN Poranga (34 ha), RPPN Angaba (29 ha) e RPPN Porangaba (9 ha), totalizando uma área conservada de 72 ha que compõem $87,8 \%$ do sítio homônimo de 82 ha, localizado na Serra do Caçador. Os limites da Reserva compreendem toda uma microbacia hidrográfica e uma pequena parte de uma microbacia hidrográfica vizinha, ambas sem denominação formal. Estas microbracias estão integralmente inseridas na Região Hidrográfica Guandu (RH II) do Estado do Rio de Janeiro e fazem parte do bloco de remanescentes florestais da Região Sul Fluminense (Rocha et al., 2003). Conforme a classificação de Köppen, o clima da região é caracterizado como AW (tropical chuvoso com inverno seco) (Valcarcel \& D’Altério, 1998). A 
temperatura máxima média anual ocorre no mês de fevereiro $\left(25^{\circ} \mathrm{C}\right)$, enquanto a mínima média anual ocorre no mês de julho $\left(19,6^{\circ} \mathrm{C}\right)$ (Valcarcel \& D’altério, 1998). A pluviosidade da região gira em torno de $1224 \mathrm{~mm} /$ ano (Brasil, 1992), com menor precipitação entre os meses de abril e setembro, variando entre 18 e $80 \mathrm{~mm}$ e maior precipitação de outubro e março, variando de 20 a $155 \mathrm{~mm}$.

O histórico de utilização da RPPN Porangaba relata o cultivo de cana-de-açúcar nas terras baixas e café nas partes mais elevadas da propriedade, posteriormente substituídos pelo cultivo da banana, que vem sendo manejado até os dias atuais. Essas ações sobre a vegetação da área provocaram a diminuição de riqueza de animais silvestres, especialmente da fauna flebotomínica (Aguiar et al., 1996). A vegetação da reserva encontra-se em processo de regeneração natural desde 1978, tendo sido tomadas algumas iniciativas de recomposição florestal nas partes mais baixas da propriedade (J. Döbereiner, comunicação pessoal).

A vegetação da RPPN Porangaba pode ser classificada como Floresta Ombrófila Densa Submontana (Veloso et al., 1991). No entanto, a área da RPPN apresenta vegetação em diferentes estádios de regeneração, com parte da área $(\sim 10 \%)$ coberta por bananais. Devido à maior dificuldade de acesso, as áreas mais preservadas da RPPN Porangaba estão localizadas nas regiões de maior elevação e com terreno mais inclinado, especialmente acima dos 300 m a.n.m. (J. Döbereiner, comunicação pessoal).

Para realizar o levantamento florístico e fitossociológico da RPPN Porangaba, foram estabelecidas parcelas fixas de $10 \times 10 \mathrm{~m}\left(100 \mathrm{~m}^{2}\right)$ (Müeller-Dombois \& Ellenberg, 1974), organizadas em 10 blocos de cinco parcelas, totalizando 50 parcelas (0,5 ha amostrados). Os blocos foram dispostos em diferentes altitudes, acompanhando-se as curvas de nível locais e as trilhas que percorrem toda a propriedade, tendo como objetivo contemplar a maior área possível da reserva. Em cada bloco, as parcelas foram estabelecidas com 10 metros de distância entre si.

Em cada parcela, foram amostrados e identificados todos os indivíduos arbóreos com DAP (diâmetro à altura do peito a $1,30 \mathrm{~m}$ do solo) igual ou superior a $5,0 \mathrm{~cm}$ e altura maior que $2,0 \mathrm{~m}$.
Todos os indivíduos amostrados tiveram o CAP (circunferência à altura do peito) mensurado (com o auxílio de fita métrica; em centímetros) e a altura estimada visualmente, tomando-se como referência uma vara de $6 \mathrm{~m}$ de altura. Posteriormente, os valores de CAP foram convertidos para DAP através da equação: $\mathrm{DAP}=\mathrm{CAP} / \pi$. Os troncos mortos ainda de pé, encontrados no interior das parcelas, foram considerados no estudo, e todos os indivíduos foram contabilizados para análises posteriores.

Foi listada a composição com base nas famílias e nas espécies amostradas na comunidade arbórea da RPPN Porangaba e calculamos a riqueza (S) e a diversidade de espécies através do índice de Shannon-Weaver (H') (Magurran, 1988). Para caracterizar a estrutura da vegetação, foram calculados os parâmetros comumente empregados em estudos fitossociológicos (Vuono, 2002) através do software Mata Nativa 2.0 (Centro..., 2006): Frequência absoluta $(\mathrm{Fa})$, Frequência relativa (FR), Frequência de ocorrência absoluta (Fo), Frequência relativa de ocorrência (Fro), Densidade absoluta (Da), Densidade relativa (DR), Área basal (G), Dominância absoluta (Doa), Dominância relativa (Dor), Valor de Cobertura (VC) e Valor de Importância (VI).

Material botânico (material vegetativo e fértil) foi coletado para posterior identificação e tratado segundo os procedimentos usuais de herborização (Mori et al., 1985). A determinação do material botânico foi realizada através de consultas a chaves analíticas, por comparação com material botânico depositado em herbários especializados ou por consulta a especialistas. A classificação do material botânico baseou-se em APG III (Souza \& Lorenzi, 2005; APG III, 2009).

\section{RESULTADOS E DISCUSSÃO}

Foi amostrado um total de 943 indivíduos, sendo 877 indivíduos vivos $(93,0 \%)$ e 66 mortos $(7,0 \%)$ (Tabela 1). Dentre os indivíduos vivos, foram registradas 105 espécies, de 69 gêneros e 36 famílias botânicas (Figura 1). As famílias mais representativas foram Fabaceae $(S=14)$, Myrtaceae $(S=11)$, Moraceae $(S=6)$, Meliaceae, Rubiaceae e Lauraceae ( $\mathrm{S}=5$ cada) (Figura 1). As 10 famílias 
mais representativas em número de espécies equivaleram a $61,0 \%$ do número total de espécies amostradas. Estudos florísticos realizados em outras áreas próximas à área estudada têm obtido resultados semelhantes quanto ao número e à representatividade das famílias amostradas (Peixoto et al., 1995; Oliveira, 2002; Peixoto et al., 2004; Conde et al., 2005).

As duas famílias mais representativas (Fabaceae e Myrtaceae) são, geralmente, as que apresentam os maiores valores de riqueza nos estudos fitossociológicos em outras áreas do Rio de Janeiro (Peixoto et al., 1995; Oliveira, 2002; Peixoto et al., 2004; Conde et al., 2005). Alguns estudos sugerem que nas áreas de encosta localizadas entre as cotas altitudinais de 50 e $500 \mathrm{~m}$, classificadas como florestas submontanas (segundo Velloso et al., 1991), exista um estoque de espécies dessas famílias, como é o caso de Fabaceae (Lima, 2000), possivelmente pela sobreposição da distribuição altitudinal de espécies com ocorrência em matas de baixada e em cotas mais elevadas (florestas montanas), fazendo com que esta área apresente, geralmente, maiores valores de riqueza. Isto destaca a importância da preservação dos remanescentes florestais nessas cotas altitudinais, já que estes poderiam conservar um conjunto de espécies representativos tanto das matas de baixada quanto das florestas submontana e montana (Peixoto et al., 2004).

A riqueza de espécies obtida para a RPPN Porangaba $(S=105)$ pode ser considerada alta quando comparada com outras áreas estudadas na região (Tabela 1), principalmente quando se analisa o critério de inclusão e a metodologia utilizada. No entanto, a curva do coletor indicou que o esforço amostral empregado neste estudo não foi suficiente para estimar a riqueza de espécies arbóreas, já que esta não alcançou uma assíntota (Figura 2), especialmente quando comparada com estimadores de riqueza de espécies (Jacknife: $\mathrm{S}=138$; Bootstrap: $S=120)$. Dessa forma, é possível que o aumento do esforço amostral possa aumentar ainda mais a riqueza de espécies, registrando especialmente aquelas espécies cujas densidades populacionais são muito baixas.

O índice de Shannon ( $\mathrm{H}^{\prime}$ ) calculado para a RPPN Porangaba apresentou o valor de 3,80 nats/indivíduo, podendo ser considerado um valor alto (Tabela 1). Esses valores corroboram a afirmação de Martins (1991) de que, em geral, os valores de diversidade obtidos para a Floresta Atlântica variam de 3,61 a 4,07. No entanto, Kurtz \& Araújo (2000) sugerem que a variação dos valores de diversidade pode ser ainda maior no Estado do Rio de Janeiro (entre 1,69

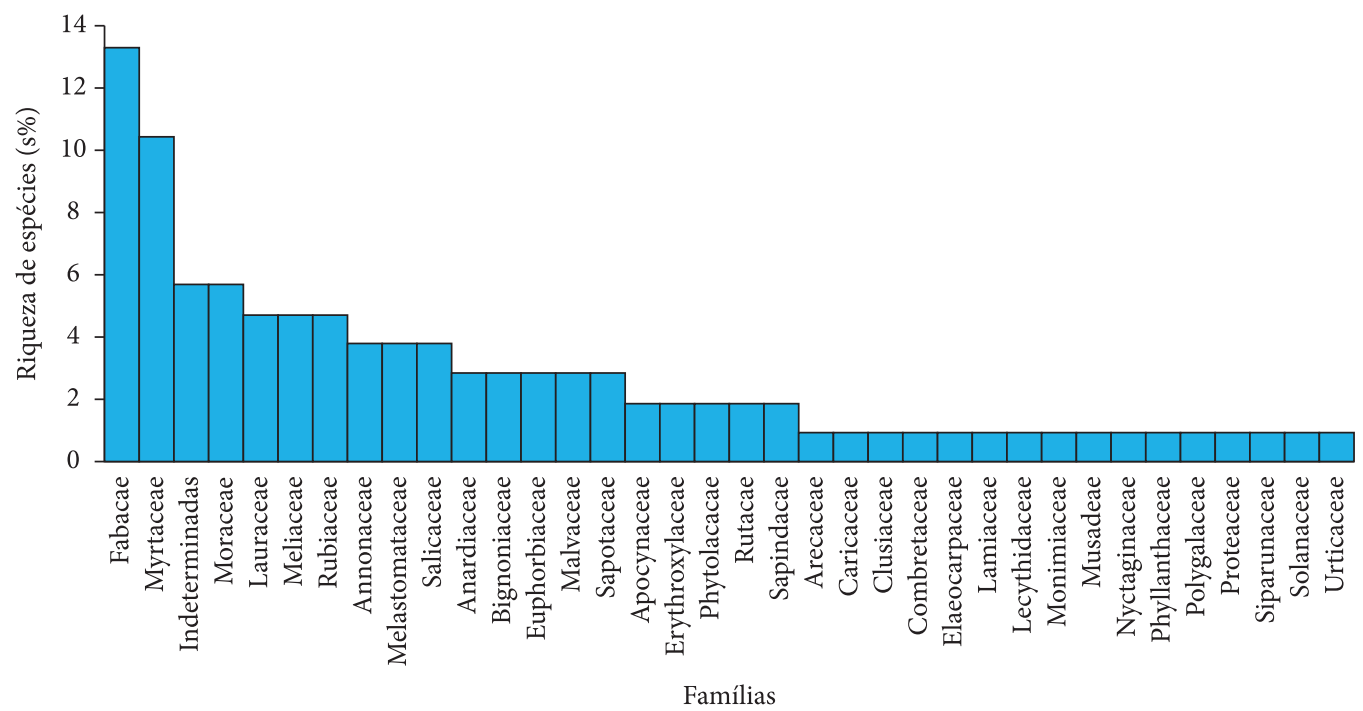

Figura 1. Representatividade percentual na riqueza de espécies (S) das famílias de espécies arbóreas registradas na RPPN Porangaba, Itaguaí, RJ.

Figure 1. Representativeness of species richness percentage $(S)$ of tree species families recorded at RPPN Porangaba, Itaguaí, RJ. 
Tabela 1. Comparação entre a riqueza (S), a diversidade de espécies (H’), área amostrada (A; em hectares) e critério de inclusão da comunidade de espécies arbóreas da RPPN Porangaba e em outras áreas de Floresta Atlântica estudadas no Estado do Rio de Janeiro.

Table 1. Comparison between richness (S), species diversity (H '), sampled area (A; in hectares) and inclusion criteria of the community tree species at RPPN Porangaba and other areas of the Atlantic Forest studied in Rio de Janeiro.

\begin{tabular}{lccccc}
\multicolumn{1}{c}{ Local } & A $\mathbf{( h a )}$ & $\begin{array}{c}\text { Critério de } \\
\text { inclusão }\end{array}$ & $\mathbf{S}$ & H' & Referência \\
\hline Ribeirão das Lajes & -- & $\mathrm{H} \geq 2,0$ & 188 & -- & Peixoto et al., 1995 \\
Marambaia & -- & -- & 175 & -- & Conde et al., 2005 \\
\hline Floresta da Cicuta & 0,3 ha & DAP $\geq 2,5$ & 184 & 3,66 & Souza, 2002 \\
Ilha Grande & 0,26 ha & DAP $\geq 2,5$ & 134 & 4,28 & Oliveira, 2002 \\
RPPN Porangaba & $\mathbf{0 , 5}$ ha & DAP $\geq \mathbf{5 , 0}$ & $\mathbf{1 0 5}$ & $\mathbf{3 , 8 0}$ & Este estudo \\
Ilha Grande & 0,26 ha & DAP $\geq 2,5$ & 70 & 3,33 & Oliveira, 2002 \\
Fazenda Atalaia & 0,3 ha & DAP $\geq 5$ & 71 & 3,94 & Cruz, dados não publicados \\
Ilha Grande & 0,26 ha & DAP $\geq 2,5$ & 63 & 3,10 & Oliveira, 2002 \\
Fazenda Atalaia & 0,3 ha & DAP $\geq 5$ & 63 & 3,58 & Cruz, dados não publicados \\
Capoeira Grande & -- & CAP $\geq 15$ & 62 & 2,42 & Peixoto et al., 2004 \\
Ilha Grande & 0,26 ha & DAP $\geq 2,5$ & 26 & 2,51 & Oliveira, 2002 \\
\hline
\end{tabular}

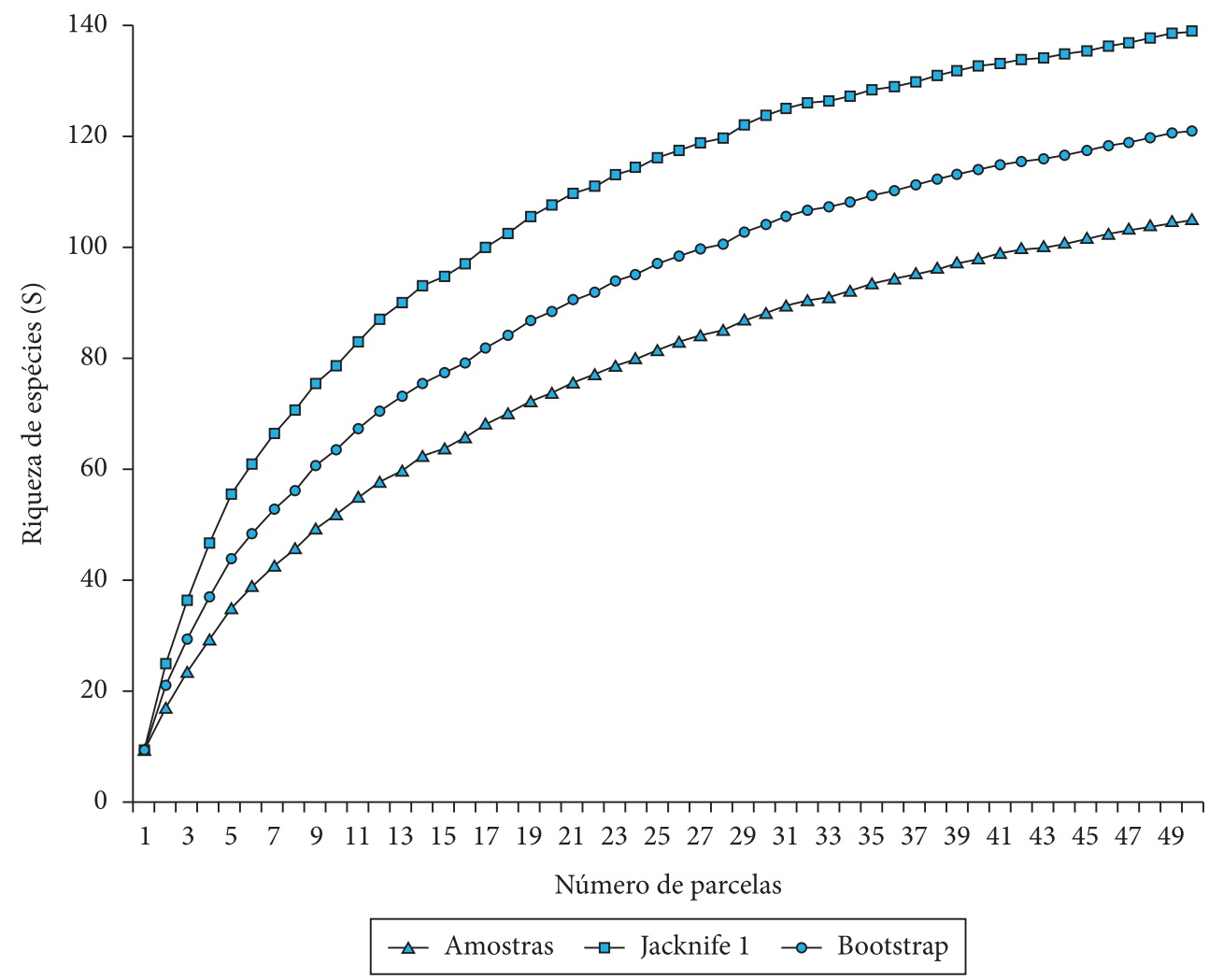

Figura 2. Curva do coletor representando o incremento de espécies arbóreas de acordo com o aumento do número de parcelas de estudo da comunidade arbórea da RPPN Porangaba, município de Itaguaí, RJ, e curvas de rarefação, obtidas através dos estimadores Jacknife 1 e Bootstrap.

Figure 2. Collector's curve representing the tree species increment according to the increase in the number of plots for the study of tree community at RPPN Porangaba, Itaguaí, RJ, and rarefaction curves of the estimators obtained through Jackknife and Bootstrap. 
e 4,4) dependendo da área analisada. Possivelmente o valor elevado de Shannon encontrado para Porangaba se deve ao fato deste índice atribuir maior peso às espécies raras (Martins, 1991), as quais foram frequentes nessa amostra (26,7\%).

Kurtz \& Araújo (2000), compilando estudos realizados na Floresta Atlântica do Estado do Rio de Janeiro, encontraram uma variação percentual de espécies raras (aquelas com apenas um indivíduo por espécie) de 9,5 a 45,2\%. O percentual encontrado para a RPPN Porangaba (45,7\%) pode ser considerado bastante elevado, já que supera o valor encontrado por esses autores. No entanto, a comparação desses percentuais deve ser vista com cuidado, uma vez que o método de estudo e o critério de inclusão utilizados, bem como o esforço amostral, influenciam tais percentuais (Kurtz \& Araújo, 2000).

Nas 50 parcelas, foram registrados 943 indivíduos, correspondendo a uma densidade total de 1886 indivíduos/ha, dos quais 877 (93,0\%) estavam vivos e 66 (7,0\%) mortos (Tabela 2). Apesar do número de indivíduos mortos ser relativamente alto, este representou uma pequena parcela do total de indivíduos amostrados, sendo semelhante ao encontrado por Oliveira (2002) em duas áreas de mata de encosta na Ilha Grande, RJ, classificadas como em estágios inicial e médio de regeneração (respectivamente 7,8 e 6,3\%). Esses resultados estão de acordo com o tempo de regeneração natural indicado para áreas com cerca de 30 anos, similar aos das áreas analisadas por Oliveira (2002), mostrando a importância da contabilização do número e do tamanho dos indivíduos mortos, já que estes são importantes para auxiliar na determinação do estágio sucessional da área analisada (Budowski, 1965; Kurtz \& Araújo, 2000).

As quatro espécies mais representativas da área de estudo foram Guarea guidonia $(\mathrm{Fa}=$ 109 indivíduos; $11,56 \%$ do total de indivíduos), Piptadenia gonoacantha $(\mathrm{Fa}=65 ; 6,89 \%)$, Casearia sylvestris $(\mathrm{Fa}=59 ; 6,26 \%)$ e Bathysa gymnocarpa $(\mathrm{Fa}=50 ; 5,3 \%)$, que somadas representam $30,1 \%$ do total de indivíduos amostrados na RPPN Porangaba (Tabela 2). Estas quatro espécies foram também as que ocorreram no maior número de parcelas na área, sendo cada uma delas registrada em mais de $50 \%$ das parcelas de amostragem (Tabela 2). Essas espécies são geralmente classificadas como espécies pioneiras ou secundárias iniciais na sucessão ecológica, apresentando elevada taxa de crescimento em suas áreas de ocorrência (e.g., Lorenzi, 2002). No entanto, é possível que estas espécies possam estar começando a sair do sistema e sendo substituídas por espécies de estágios mais tardios, mas ainda são necessários estudos de dinâmica de suas populações para que isso seja mais bem compreendido.

$\mathrm{O}$ valor de área basal da vegetação da RPPN Porangaba $\left(\mathrm{G}=20,71 \mathrm{~m}^{2} /\right.$ ha para 943 indivíduos amostrados) pode ser considerado baixo quando comparado com outros estudos em diferentes regiões do Estado do Rio de Janeiro. Cruz (dados não publicados), estudando duas áreas no Parque Natural Municipal Fazenda Atalaia, em Macaé, obteve valores de área basal de $26,41 \mathrm{~m}^{2} / \mathrm{ha}$ (404 indivíduos) e $35,59 \mathrm{~m}^{2} / \mathrm{ha}$ (231 indivíduos). Oliveira (2002), estudando quatro áreas na Ilha Grande, encontrou valores entre 5,6 e 57,9 $\mathrm{m}^{2} /$ ha para um total de 2332 indivíduos. Kurtz \& Araújo (2000), estudando uma vegetação climáxica (ou em estágio sucessional próximo) na Estação Ecológica Estadual do Paraíso (Cachoeiras de Macacu), obteve para 592 indivíduos amostrados uma área basal de $57,28 \mathrm{~m}^{2} / \mathrm{ha}$. Silva \& Nascimento (2001), em um remanescente de mata de tabuleiro em São Francisco do Itabapoana, amostrou um total de 564 indivíduos e o equivalente a uma área basal de $15,0 \mathrm{~m}^{2} / \mathrm{ha}$, enquanto Pessoa et al. (1997) encontraram 2217 indivíduos e uma área basal de $27,95 \mathrm{~m}^{2} / \mathrm{ha}$ em um trecho de floresta montana em estágio secundária. Esses dados sugerem que apesar do tempo em que a área se encontra em sucessão espontânea, ela ainda pode ser considerada como em estágio variando entre o inicial e o médio de regeneração.

As espécies que mais contribuíram para área basal e, consequentemente, apresentaram os maiores valores de dominância foram Piptadenia gonoacantha $\left(\mathrm{G}=4,00 \mathrm{~m}^{2} ;\right.$ Doa $\left.=8,00 \mathrm{~m}^{2} / \mathrm{ha}\right)$, G. guidonea $(\mathrm{G}=$ $2,85 \mathrm{~m}^{2}$; Doa $\left.=5,70 \mathrm{~m}^{2} / \mathrm{ha}\right)$, as árvores mortas $(\mathrm{G}=$ $1,72 \mathrm{~m}^{2}$; Doa $\left.=3,45 \mathrm{~m}^{2} / \mathrm{ha}\right)$ e Apuleia leiocarpa $(\mathrm{G}=$ $1,29 \mathrm{~m}^{2}$; Doa $=2,57 \mathrm{~m}^{2} /$ ha) (Tabela 2). Essas espécies contribuíram com $9,86 \mathrm{~m}^{2}$, o equivalente a $47,61 \%$ de área basal total, e uma dominância relativa de $63,3 \%$ (Tabela 2). 
Tabela 2. Parâmetros fitossociológicos da comunidade arbórea na RPPN Porangaba, Itaguaí, RJ. Fa = frequência absoluta; Fr = frequência relativa; Fo = frequência absoluta de ocorrência nas parcelas de amostragem $(\%)$; Fro = frequência relativa de ocorrência nas parcelas de amostragem (\%); Da = densidade absoluta (indivíduos/ha); Dr = densidade relativa (\%); $\mathrm{G}=$ área basal $\left(\mathrm{m}^{2}\right) ; \mathrm{DoA}=$ dominância absoluta $\left(\mathrm{m}^{2} / \mathrm{ha}\right) ; \mathrm{DoR}=$ dominância relativa $(\%)$; $\mathrm{VC}=$ valor de cobertura (\%); VI = valor de importância (\%).

Table 2. Phytosociological parameters of the tree community at RPPN Porangaba, Itaguaí, RJ. Fa = absolute frequency; Fr = relative frequency; Fo = absolute frequency of occurrence in plots of sampling $(\%)$; Fro = relative frequency of occurrence in plots of sampling (\%); $\mathrm{Da}=$ absolute density (individual trees $/ \mathrm{ha}$ ), $\mathrm{Dr}=$ relative density (\%), $\mathrm{G}=$ basal area $\left(\mathrm{m}^{2}\right) ; \mathrm{DoA}=$ absolute dominance $\left(\mathrm{m}^{2} / \mathrm{ha}\right) ; \mathrm{DoR}=$ relative dominance $(\%) ; \mathrm{VC}=\operatorname{cover}(\%)$; VI = importance value $(\%)$.

\begin{tabular}{|c|c|c|c|c|c|c|c|c|c|c|c|}
\hline Espécie & $\mathrm{Fa}$ & Fr & Fo & Fro & Da & Dr & G & Doa & Dor & VC & VI \\
\hline Guarea guidonia (L.) Sleumer & 109 & 11,56 & 33 & 66 & 218 & 11,56 & 2,84817 & 5,70 & 13,75 & 25,31 & 36,87 \\
\hline Piptadenia gonoacantha (Mart.) J.F. Macbr & 65 & 6,89 & 26 & 52 & 130 & 6,89 & 4,00094 & 8,00 & 19,32 & 26,21 & 33,10 \\
\hline Morta & 66 & 7,00 & 36 & 72 & 132 & 7,00 & 1,72325 & 3,45 & 8,32 & 15,32 & 22,32 \\
\hline Casearia sylvestris $\mathrm{Sw}$. & 59 & 6,26 & 25 & 50 & 118 & 6,26 & 0,61167 & 1,22 & 2,95 & 9,21 & 15,47 \\
\hline Bathysa gymnocarpa K. Schum. & 50 & 5,30 & 11 & 22 & 100 & 5,30 & 0,36713 & 0,73 & 1,77 & 7,07 & 12,38 \\
\hline Erythroxylum pulchrum A. St.-Hill. & 38 & 4,03 & 15 & 30 & 76 & 4,03 & 0,54304 & 1,09 & 2,62 & 6,65 & 10,68 \\
\hline Apuleia leiocarpa (Vogel) J.F. Macbr. & 17 & 1,80 & 12 & 24 & 34 & 1,80 & 1,28741 & 2,57 & 6,22 & 8,02 & 9,82 \\
\hline Astrocaryum aculeatissimum (Schott) Burret & 34 & 3,61 & 11 & 22 & 68 & 3,61 & 0,28237 & 0,56 & 1,36 & 4,97 & 8,57 \\
\hline Galipea laxiflora Engl. & 32 & 3,39 & 16 & 32 & 64 & 3,39 & 0,23833 & 0,48 & 1,15 & 4,54 & 7,94 \\
\hline $\begin{array}{l}\text { Pseudopiptadenia contorta (DC.) G.P. Lewis \& M.P. } \\
\text { Lima }\end{array}$ & 23 & 2,44 & 14 & 28 & 46 & 2,44 & 0,6059 & 1,21 & 2,93 & 5,36 & 7,80 \\
\hline Lauraceae sp.1 & 24 & 2,55 & 13 & 26 & 48 & 2,55 & 0,32425 & 0,65 & 1,57 & 4,11 & 6,66 \\
\hline Machaerium hirtum (Vell.) Stellfeld & 17 & 1,80 & 9 & 18 & 34 & 1,80 & 0,62603 & 1,25 & 3,02 & 4,83 & 6,63 \\
\hline Nectandra membranacea (SW.) Griseb. & 19 & 2,01 & 16 & 32 & 38 & 2,01 & 0,49612 & 0,99 & 2,40 & 4,41 & 6,43 \\
\hline Pterocarpus violaceus Vogel & 10 & 1,06 & 8 & 16 & 20 & 1,06 & 0,86424 & 1,73 & 4,17 & 5,23 & 6,29 \\
\hline Guapira opposita (Vell.) Reitz & 23 & 2,44 & 13 & 26 & 46 & 2,44 & 0,18969 & 0,38 & 0,92 & 3,35 & 5,79 \\
\hline Luehea grandiflora Mart. & 16 & 1,70 & 11 & 22 & 32 & 1,70 & 0,13743 & 0,27 & 0,66 & 2,36 & 4,06 \\
\hline Cupania oblongifolia Mart. & 16 & 1,70 & 11 & 22 & 32 & 1,70 & 0,1326 & 0,27 & 0,64 & 2,34 & 4,03 \\
\hline Joannesia princeps Vell. & 8 & 0,85 & 6 & 12 & 16 & 0,85 & 0,4572 & 0,91 & 2,21 & 3,06 & 3,90 \\
\hline Casearia obliqua Spreng. & 13 & 1,38 & 4 & 8 & 26 & 1,38 & 0,20213 & 0,40 & 0,98 & 2,35 & 3,73 \\
\hline Casearia aff. sessiliflora Cambess & 15 & 1,59 & 10 & 20 & 30 & 1,59 & 0,10964 & 0,22 & 0,53 & 2,12 & 3,71 \\
\hline Annona cacans Warm. & 5 & 0,53 & 4 & 8 & 10 & 0,53 & 0,44363 & 0,89 & 2,14 & 2,67 & 3,20 \\
\hline Sloanea sp. & 9 & 0,95 & 7 & 14 & 18 & 0,95 & 0,25503 & 0,51 & 1,23 & 2,19 & 3,14 \\
\hline Sparattosperma leucanthum (Vell.) K. Schum. & 11 & 1,17 & 9 & 18 & 22 & 1,17 & 0,16185 & 0,32 & 0,78 & 1,95 & 3,11 \\
\hline Psychotria brasiliensis Vell. & 10 & 1,06 & 6 & 12 & 20 & 1,06 & 0,12854 & 0,26 & 0,62 & 1,68 & 2,74 \\
\hline Chrysophyllum flexuosum Mart. & 11 & 1,17 & 5 & 10 & 22 & 1,17 & 0,08108 & 0,16 & 0,39 & 1,56 & 2,72 \\
\hline Astronium fraxinifolium Schott ex Spreng. & 9 & 0,95 & 9 & 18 & 18 & 0,95 & 0,1401 & 0,28 & 0,68 & 1,63 & 2,59 \\
\hline Jacaratia spinosa (Aubl.) A. DC. & 4 & 0,42 & 4 & 8 & 8 & 0,42 & 0,31496 & 0,63 & 1,52 & 1,94 & 2,37 \\
\hline Trichilia hirta L. & 9 & 0,95 & 3 & 6 & 18 & 0,95 & 0,09235 & 0,18 & 0,45 & 1,40 & 2,35 \\
\hline $\begin{array}{l}\text { Hymenaea courbaril var. stilbocarpa (Hayne) Y.T. } \\
\text { Lee \& Langenh. }\end{array}$ & 8 & 0,85 & 7 & 14 & 16 & 0,85 & 0,10854 & 0,22 & 0,52 & 1,37 & 2,22 \\
\hline Cedrela fissilis Vell. & 8 & 0,85 & 6 & 12 & 16 & 0,85 & 0,10871 & 0,22 & 0,52 & 1,37 & 2,22 \\
\hline Nectandra grandiflora Nees \& C. Mart. ex Nees & 8 & 0,85 & 5 & 10 & 16 & 0,85 & 0,09431 & 0,19 & 0,46 & 1,30 & 2,15 \\
\hline Citrus reticulata Blanco & 8 & 0,85 & 3 & 6 & 16 & 0,85 & 0,05787 & 0,12 & 0,28 & 1,13 & 1,98 \\
\hline Hyeronima aff. alchorneoides Allemão & 6 & 0,64 & 3 & 6 & 12 & 0,64 & 0,12456 & 0,25 & 0,60 & 1,24 & 1,87 \\
\hline Inga laurina (Sw.) Wild. & 6 & 0,64 & 2 & 4 & 12 & 0,64 & 0,1182 & 0,24 & 0,57 & 1,21 & 1,84 \\
\hline Gallesia integrifolia (Spreng.) Harms & 3 & 0,32 & 3 & 6 & 6 & 0,32 & 0,22674 & 0,45 & 1,09 & 1,41 & 1,73 \\
\hline Machaerium nyctitans (Vell.) Benth. & 7 & 0,74 & 6 & 12 & 14 & 0,74 & 0,03206 & 0,06 & 0,15 & 0,90 & 1,64 \\
\hline Piptadenia paniculata Benth. & 6 & 0,64 & 5 & 10 & 12 & 0,64 & 0,0483 & 0,10 & 0,23 & 0,87 & 1,51 \\
\hline Roupala meisneri Sleumer & 6 & 0,64 & 3 & 6 & 12 & 0,64 & 0,04604 & 0,09 & 0,22 & 0,86 & 1,49 \\
\hline Chrysophyllum splendens Spreng. & 6 & 0,64 & 2 & 4 & 12 & 0,64 & 0,04483 & 0,09 & 0,22 & 0,85 & 1,49 \\
\hline Chorisia speciosa A. St.-Hil & 6 & 0,64 & 3 & 6 & 12 & 0,64 & 0,03178 & 0,06 & 0,15 & 0,79 & 1,43 \\
\hline Mimosoideae sp.1 & 2 & 0,21 & 1 & 2 & 4 & 0,21 & 0,18857 & 0,38 & 0,91 & 1,12 & 1,33 \\
\hline Trichilia elegans A.Juss. & 5 & 0,53 & 4 & 8 & 10 & 0,53 & 0,04603 & 0,09 & 0,22 & 0,75 & 1,28 \\
\hline
\end{tabular}


Tabela 2. Continuação...

Table 2. Continued...

\begin{tabular}{|c|c|c|c|c|c|c|c|c|c|c|c|}
\hline Espécie & $\mathbf{F a}$ & Fr & Fo & Fro & Da & Dr & G & Doa & Dor & VC & VI \\
\hline Miconia prasina DC & 5 & 0,53 & 5 & 10 & 10 & 0,53 & 0,04207 & 0,08 & 0,20 & 0,73 & 1,26 \\
\hline Combretaceae sp. 1 & 4 & 0,42 & 3 & 6 & 8 & 0,42 & 0,08329 & 0,17 & 0,40 & 0,83 & 1,25 \\
\hline Tabebuia heptaphylla (Vell.) Toledo & 5 & 0,53 & 3 & 6 & 10 & 0,53 & 0,03166 & 0,06 & 0,15 & 0,68 & 1,21 \\
\hline Cariniana legalis (Mart.) Kuntze & 2 & 0,21 & 2 & 4 & 4 & 0,21 & 0,15693 & 0,31 & 0,76 & 0,97 & 1,18 \\
\hline Sapium glandulatum (Vell.) Pax & 4 & 0,42 & 4 & 8 & 8 & 0,42 & 0,0667 & 0,13 & 0,32 & 0,75 & 1,17 \\
\hline Rubiaceae sp.1 & 5 & 0,53 & 1 & 2 & 10 & 0,53 & 0,01455 & 0,03 & 0,07 & 0,60 & 1,13 \\
\hline Trichilia casaretti C.DC. & 5 & 0,53 & 3 & 6 & 10 & 0,53 & 0,01347 & 0,03 & 0,07 & 0,60 & 1,13 \\
\hline Swartzia langsdorffii Raddi & 5 & 0,53 & 2 & 4 & 10 & 0,53 & 0,01302 & 0,03 & 0,06 & 0,59 & 1,12 \\
\hline Seguieria langsdorffii Moq. & 1 & 0,11 & 1 & 2 & 2 & 0,11 & 0,18144 & 0,36 & 0,88 & 0,98 & 1,09 \\
\hline Caesalpinioideae sp.1 & 3 & 0,32 & 2 & 4 & 6 & 0,32 & 0,0863 & 0,17 & 0,42 & 0,73 & 1,05 \\
\hline Myrcia rostrata DC. & 3 & 0,32 & 3 & 6 & 6 & 0,32 & 0,07143 & 0,14 & 0,34 & 0,66 & 0,98 \\
\hline Miconia calvescens DC. & 4 & 0,42 & 3 & 6 & 8 & 0,42 & 0,02398 & 0,05 & 0,12 & 0,54 & 0,96 \\
\hline Sapindaceae sp.1 & 4 & 0,42 & 4 & 8 & 8 & 0,42 & 0,02274 & 0,05 & 0,11 & 0,53 & 0,96 \\
\hline Astronium graveolens Jacq. & 4 & 0,42 & 3 & 6 & 8 & 0,42 & 0,01965 & 0,04 & 0,09 & 0,52 & 0,94 \\
\hline $\begin{array}{l}\text { Sorocea bonplandii (Baill.) W.C. Burger, Lanj. \& } \\
\text { Wess. Boer }\end{array}$ & 3 & 0,32 & 3 & 6 & 6 & 0,32 & 0,06173 & 0,12 & 0,30 & 0,62 & 0,93 \\
\hline Casearia commersoniana Cambess. & 3 & 0,32 & 3 & 6 & 6 & 0,32 & 0,0427 & 0,09 & 0,21 & 0,52 & 0,84 \\
\hline Caesalpinia ferrea var. leiostachya Benth. & 1 & 0,11 & 1 & 2 & 2 & 0,11 & 0,11844 & 0,24 & 0,57 & 0,68 & 0,78 \\
\hline Gomidesia tijucensis (Kiaersk.) D. Legrand & 3 & 0,32 & 3 & 6 & 6 & 0,32 & 0,02554 & 0,05 & 0,12 & 0,44 & 0,76 \\
\hline Pouteria caimito (Ruiz \& Pav.) Radlk. & 3 & 0,32 & 3 & 6 & 6 & 0,32 & 0,02274 & 0,05 & 0,11 & 0,43 & 0,75 \\
\hline Solanum sp. & 3 & 0,32 & 2 & 4 & 6 & 0,32 & 0,01046 & 0,02 & 0,05 & 0,37 & 0,69 \\
\hline Eugenia oblongata O. Berg. & 3 & 0,32 & 3 & 6 & 6 & 0,32 & 0,01007 & 0,02 & 0,05 & 0,37 & 0,68 \\
\hline Peschiera affinis (Müll. Arg.) Miers & 3 & 0,32 & 2 & 4 & 6 & 0,32 & 0,00972 & 0,02 & 0,05 & 0,37 & 0,68 \\
\hline Eugenia candolleana DC. & 3 & 0,32 & 2 & 4 & 6 & 0,32 & 0,00946 & 0,02 & 0,05 & 0,36 & 0,68 \\
\hline Sorocea hilarii Gaudich. & 3 & 0,32 & 3 & 6 & 6 & 0,32 & 0,00665 & 0,01 & 0,03 & 0,35 & 0,67 \\
\hline Morfoespécie 03 & 1 & 0,11 & 1 & 2 & 2 & 0,11 & 0,08773 & 0,18 & 0,42 & 0,53 & 0,64 \\
\hline Ficus arpazusa Casar. & 2 & 0,21 & 1 & 2 & 4 & 0,21 & 0,03165 & 0,06 & 0,15 & 0,36 & 0,58 \\
\hline Artocarpus heterophyllus Lam. & 1 & 0,11 & 1 & 2 & 2 & 0,11 & 0,07334 & 0,15 & 0,35 & 0,46 & 0,57 \\
\hline Marlieria sp. & 2 & 0,21 & 2 & 4 & 4 & 0,21 & 0,02873 & 0,06 & 0,14 & 0,35 & 0,56 \\
\hline Xylopia brasiliensis Spreng. & 2 & 0,21 & 1 & 2 & 4 & 0,21 & 0,02608 & 0,05 & 0,13 & 0,34 & 0,55 \\
\hline Brosimum guianensis (Aubl.) Huber & 2 & 0,21 & 2 & 4 & 4 & 0,21 & 0,02254 & 0,05 & 0,11 & 0,32 & 0,53 \\
\hline Ficus insipida Wild. & 2 & 0,21 & 1 & 2 & 4 & 0,21 & 0,01755 & 0,04 & 0,08 & 0,30 & 0,51 \\
\hline Clusia sp. & 2 & 0,21 & 2 & 4 & 4 & 0,21 & 0,01569 & 0,03 & 0,08 & 0,29 & 0,50 \\
\hline Aspidosperma sp. & 2 & 0,21 & 2 & 4 & 4 & 0,21 & 0,0136 & 0,03 & 0,07 & 0,28 & 0,49 \\
\hline Myrtaceae sp.1 & 2 & 0,21 & 2 & 4 & 4 & 0,21 & 0,01175 & 0,02 & 0,06 & 0,27 & 0,48 \\
\hline Eugenia sp. & 1 & 0,11 & 1 & 2 & 2 & 0,11 & 0,05482 & 0,11 & 0,26 & 0,37 & 0,48 \\
\hline Annonaceae sp. 1 & 2 & 0,21 & 2 & 4 & 4 & 0,21 & 0,01045 & 0,02 & 0,05 & 0,26 & 0,47 \\
\hline Jacaranda aff. Puberula Cham. & 2 & 0,21 & 2 & 4 & 4 & 0,21 & 0,00755 & 0,02 & 0,04 & 0,25 & 0,46 \\
\hline Morfoespécie 06 & 2 & 0,21 & 2 & 4 & 4 & 0,21 & 0,00739 & 0,01 & 0,04 & 0,25 & 0,46 \\
\hline Psychotria mapourioides DC. & 2 & 0,21 & 2 & 4 & 4 & 0,21 & 0,00669 & 0,01 & 0,03 & 0,24 & 0,46 \\
\hline Erythroxylum sp. & 2 & 0,21 & 2 & 4 & 4 & 0,21 & 0,00581 & 0,01 & 0,03 & 0,24 & 0,45 \\
\hline Morfoespécie 05 & 2 & 0,21 & 1 & 2 & 4 & 0,21 & 0,0046 & 0,01 & 0,02 & 0,23 & 0,45 \\
\hline Morfoespécie 02 & 1 & 0,11 & 1 & 2 & 2 & 0,11 & 0,03259 & 0,07 & 0,16 & 0,26 & 0,37 \\
\hline Morfoespécie 01 & 1 & 0,11 & 1 & 2 & 2 & 0,11 & 0,02677 & 0,05 & 0,13 & 0,24 & 0,34 \\
\hline Nectandra oppositifolia Ness & 1 & 0,11 & 1 & 2 & 2 & 0,11 & 0,02407 & 0,05 & 0,12 & 0,22 & 0,33 \\
\hline Morfoespécie 04 & 1 & 0,11 & 1 & 2 & 2 & 0,11 & 0,0207 & 0,04 & 0,10 & 0,21 & 0,31 \\
\hline Miconia brasiliensis Trian. & 1 & 0,11 & 1 & 2 & 2 & 0,11 & 0,01338 & 0,03 & 0,06 & 0,17 & 0,28 \\
\hline Inga sp. & 1 & 0,11 & 1 & 2 & 2 & 0,11 & 0,0121 & 0,02 & 0,06 & 0,16 & 0,27 \\
\hline Miconia cinnamomifolia (DC.) Naudin & 1 & 0,11 & 1 & 2 & 2 & 0,11 & 0,01031 & 0,02 & 0,05 & 0,16 & 0,26 \\
\hline Musa sapientum $\mathrm{L}$. & 1 & 0,11 & 1 & 2 & 2 & 0,11 & 0,00669 & 0,01 & 0,03 & 0,14 & 0,24 \\
\hline Rollinia cf. dollabripetala (Raddi) R.E.Fr. & 1 & 0,11 & 1 & 2 & 2 & 0,11 & 0,00669 & 0,01 & 0,03 & 0,14 & 0,24 \\
\hline
\end{tabular}


Tabela 2. Continuação...

Table 2. Continued...

\begin{tabular}{lccccccccccc}
\multicolumn{1}{c}{ Espécie } & Fa & Fr & Fo & Fro & Da & Dr & G & Doa & Dor & VC & VI \\
\hline Eugenia tinguyensis Cambess. & 1 & 0,11 & 1 & 2 & 2 & 0,11 & 0,00624 & 0,01 & 0,03 & 0,14 & 0,24 \\
Quararibea turbinata (Sw.) Poir. & 1 & 0,11 & 1 & 2 & 2 & 0,11 & 0,00497 & 0,01 & 0,02 & 0,13 & 0,24 \\
Aegiphyla sellowiana Cham. & 1 & 0,11 & 1 & 2 & 2 & 0,11 & 0,00458 & 0,01 & 0,02 & 0,13 & 0,23 \\
Mollinedia oligantha Perkins & 1 & 0,11 & 1 & 2 & 2 & 0,11 & 0,00421 & 0,01 & 0,02 & 0,13 & 0,23 \\
Eugenia rostrata O.Berg. & 1 & 0,11 & 1 & 2 & 2 & 0,11 & 0,00318 & 0,01 & 0,02 & 0,12 & 0,23 \\
Psychotria nuda (Cham. \& Schltdl.) Wawra & 1 & 0,11 & 1 & 2 & 2 & 0,11 & 0,00318 & 0,01 & 0,02 & 0,12 & 0,23 \\
Snefeldera verticilata Vell. Croizat & 1 & 0,11 & 1 & 2 & 2 & 0,11 & 0,00318 & 0,01 & 0,02 & 0,12 & 0,23 \\
Coussapoa sp. & 1 & 0,11 & 1 & 2 & 2 & 0,11 & 0,00258 & 0,01 & 0,01 & 0,12 & 0,22 \\
Thyrsodium spruceanum Salzm. ex Benth. & 1 & 0,11 & 1 & 2 & 2 & 0,11 & 0,00258 & 0,01 & 0,01 & 0,12 & 0,22 \\
Coccoloba sp. & 1 & 0,11 & 1 & 2 & 2 & 0,11 & 0,0023 & 0,00 & 0,01 & 0,12 & 0,22 \\
\hline Eugenia tenuifolia O.Berg & 1 & 0,11 & 1 & 2 & 2 & 0,11 & 0,0023 & 0,00 & 0,01 & 0,12 & 0,22 \\
Ocotea pulchella (Ness) Mez & 1 & 0,11 & 1 & 2 & 2 & 0,11 & 0,0023 & 0,00 & 0,01 & 0,12 & 0,22 \\
Siparuna guianensis Aubl. & 1 & 0,11 & 1 & 2 & 2 & 0,11 & 0,0023 & 0,00 & 0,01 & 0,12 & 0,22 \\
Myrciaria trunciflora Mattos & 1 & 0,11 & 1 & 2 & 2 & 0,11 & 0,00204 & 0,00 & 0,01 & 0,12 & 0,22 \\
Total geral & 943 & 100 & & & 1886 & 100 & 20,7116 & 41,4232 & 100,0 & 200,0 & 300,0 \\
\hline
\end{tabular}

Em geral, os dados indicaram que apenas um pequeno número dentre as espécies amostradas na RPPN Porangaba apresentaram valores elevados de VC e VI (quatro espécies somadas à classe de indivíduos mortos), padrão esse típico das florestas tropicais (Martins, 1991; Borém \& OliveiraFilho, 2002). As espécies com os maiores valores de cobertura e de valor de importância também foram $P$. gonoacantha $(\mathrm{VC}=26,21 ; \mathrm{VI}=33,10) \mathrm{e}$ G. guidonia ( $\mathrm{VC}=25,31 ; \mathrm{VI}=36,87$ ) (Tabela 2). Essas duas espécies são citadas em outros estudos como as espécies que geralmente mais contribuem com as comunidades arbóreas de florestas secundárias, seja por serem típicas emergentes do dossel, como P. gonoacantha (Borém \& OliveiraFilho, 2002) ou por contribuírem com um elevado número de indivíduos, como é o caso de G. guidonia (Carvalho et al., 2007).

Os dados do presente estudo indicaram que a RPPN Porangaba apresenta uma riqueza de espécies elevada em relação a outras áreas estudadas no Estado do Rio de Janeiro, o que pode ser explicado pelo efeito do esforço amostral e da classe de inclusão de DAP. As famílias amostradas são as tipicamente encontradas em outros estudos, em especial aquelas que apresentam maior representatividade na amostra, o que pode ser uma resposta da distribuição altitudinal de espécies que ocorrem abaixo (nas matas de baixada) ou acima da cota amostrada (nas florestas montanas), sobrepondo suas distribuições nas florestas submontanas ou de encosta. Os valores obtidos para os parâmetros fitossociológicos também corroboram os resultados de outros estudos e classificam a área como uma área importante para conservação, especialmente pelo tempo de regeneração (entre 25 e 50 anos), guardando uma diversidade biológica representativa da formação estudada. No entanto, estudos com a dinâmica das populações das espécies registradas na área podem elucidar alguns dos resultados observados, especialmente para compreender a dinâmica da comunidade vegetal da RPPN Porangaba.

\section{AGRADECIMENTOS}

Ao Dr. Jurgen Döbereiner, proprietário da RPPN Porangaba, pela autorização para a realização deste estudo. À Alexandra Pires e Thereza Christina RochaPessôa pelas importantes contribuições na leitura do manuscrito. Este estudo fez parte do Projeto de Pesquisa "Estrutura e dinâmica de comunidades vegetais em fragmentos de Floresta Atlântica do Rio de Janeiro" e foi parcialmente subvencionado com Auxílio Instalação da Fundação Carlos Chagas Filho de Amparo à Pesquisa do Estado do Rio de Janeiro FAPERJ (Processo No E-26/170.238/2006). Durante 
o estudo M.F. Gandra recebeu bolsa do Programa Interno de Bolsa de Iniciação Científica (PROIC/ DPPG/UFRuralRJ) e M. Schütte recebeu bolsa de mestrado da Coordenação de Aperfeiçoamento de Pessoal de Nível Superior (CAPES).

\section{STATUS DA SUBMISSÃO}

Recebido: 07/06/2010

Aceito: 05/03/2011

Resumo publicado online: 13/03/2011

Artigo completo publicado: 31/03/2011

\section{AUTOR (ES) PARA CORRESPONDÊNCIA}

\section{André Felippe Nunes-Freitas}

Laboratório de Ecologia Florestal e Biologia Vegetal,

Departamento de Ciências Ambientais, Instituto de Florestas,

Universidade Federal Rural do Rio de Janeiro,

Rod. BR-465, Km 7, CEP 23890-000,

Seropédica, RJ, Brasil

e-mail: afnfreitas@ufrrj.br

\section{REFERÊNCIAS}

Aguiar GM, Medeiros WM, De Marco TS, Santos SC, Gambardella S. Ecologia dos flebotomídeos da Serra do Mar, Itaguaí, Estado do Rio de Janeiro, Brasil. Cadernos de Saúde Pública 1996; 12(2):195-206. http://dx.doi. org/10.1590/S0102-311X1996000200008[

APG - Angiosperm Phylogeny Group. An update of the Angiosperm Phylogeny Group classification for the orders and families of flowering plants: APG III. Botanical Journal of the Linnean Society 2009; 161(2):105-121.

Brasil. Ministério da Agricultura e Reforma Agrária MARA. Secretaria Nacional de Irrigação, Departamento Nacional de Meteorologia. Normais Climatológicas (1961-1990). Brasília; 1992. 84 p.

Borém RAT, Oliveira-Filho AT. Fitossociologia do estrato arbóreo em uma topossequência alterada de Mata Atlântica, no município de Silva Jardim - RJ, Brasil. Revista Árvore 2002; 26(6):727-742.

Budowsk G. Distribution of Tropical Rain Forest trees in the light of sucessional process. Turrialba 1965; 15:40-45.
Carvalho FA, Nascimento MT, Braga JMA. Estrutura e composição florística do estrato arbóreo de um remanescente de Mata Atlântica submontana no município de Rio Bonito, RJ, Brasil (Mata Rio Vermelho). Revista Árvore 2007; 31(4)717-730.

Centro integrado de Ensino Técnico - Cientec. Software Mata Nativa 2: Sistema para análise estatística e fitossociológica, laboração de inventários e Planos de Manejo de Florestas Nativas. Versão 2.0X. Viçosa: Cientec Ltda.; 2006.

Conde MMS, Lima HRP, Peixoto AL. Aspectos florísticos e vegetacionais da Marambaia, Rio de Janeiro, Brasil. In: Menezes LFT, Peixoto AL, Araújo DSD, editores. História Natural da Marambaia. Seropédica: EDUR; 2005. p. 133-168.

Dean W. A Ferro e fogo: a história e a devastação da Mata Atlântica brasileira. São Paulo: Companhia das Letras; 1997.484 p.

Fundação Sos Mata Atlântica/Inpe. Atlas dos remanescentes florestais da Mata Atlântica período 20002005. São Paulo: Fundação SOS Mata Atlântica/INPE; 2006.

Kurtz BC, Araújo DSD. Composição florística e estrutura do componente arbóreo de um trecho de Mata Atlântica na Estação Ecológica Estadual do Paraíso, Cachoeiras de Macacu, Rio de Janeiro, Brasil. Rodriguésia 2000; 51(78/115);69-112.

Lima HC. Leguminosas arbóreas da Mata Atlântica. Uma análise de riqueza, padrões de distribuição geográfica e similaridades florísticas em remanescentes florestais do estado do Rio de Janeiro [tese]. Rio de janeiro: Universidade Federal do Rio de Janeiro; 2000.

Lorenzi H. Árvores Brasileiras: manual de identificação e cultivo de plantas arbóreas nativas do Brasil. $4^{\text {rd }}$ ed. Nova Odessa: Instituto Plantarum, 2002. 382 p. vol. 1.

Magurran AE. Ecological Diversity and Its Measurement. Cambridge: University Press; 1988. 179 p.

Martins FR. Estrutura de uma floresta mesófila. $2^{\text {rd }}$ ed. Campinas: Ed. da UNICAMP 1991. 246 p. Série Teses.

Morellato LPC, Haddad CFB. Introduction: The Brazilian Atlantic Forest. Biotropica 2000; 32(4B):786792.

Mori SA, Silva LAM, Lisboa G, Coradin L. Manual de manejo de herbário fanerogâmico. Ilhéus: CEPLAC, 1985. $104 \mathrm{p}$.

Müeller-Dombois D, Ellenberg H. 1974. Aims and methods of vegetation ecology. New York: John Wiley \& Sons; 1974.547 p. 
Oliveira RR. Ação antrópica e resultantes sobre a estrutura e composição da mata atlântica na Ilha Grande, RJ. Rodriguésia 2002; 53(82):33-58.

Peixoto AL, Rosa MMT, Barbosa MRV, Rodrigues HC. Composição Florística da área em torno da represa de Ribeirão das Lajes, Rio de Janeiro, Brasil. Revista da Universidade Rural, Série Ciências da Vida 1995; 17(1)51-74.

Peixoto AL, Rosa MMT, Silva IM. Caracterização da Mata Atlântica. In: Sylvestre LS, Rosa MMT, organizadores. Manual metodológico para estudos botânicos na Mata Atlântica. Seropédica: Editora Universidade Rural; 2002. p. 9-23.

Peixoto GL, Martins SV, Silva AF, Silva E. Composição florística do componente arbóreo de um trecho de Floresta Atlântica na área de Proteção Ambiental da Serra da Capoeira Grande, Rio de Janeiro, RJ, Brasil. Acta Botânica Brasílica 2004; 18(1):151-160.

Pessoa SVA, Guedes-Bruni RR, Kurtz BC. Composição florística e estrutura do componente arbustivo-arbóreo de um trecho secundário de Floresta Montana na Reserva Ecológica de Macaé de Cima. In: Lima HC, Guedes-Bruni RR, organizadores. Serra de Macaé de Cima: Diversidade florística e Conservação em Mata Atlântica. Rio de Janeiro: Jardim Botânico do Rio de Janeiro; 1997. p. 147-168.

Rambaldi DM, Magnani A, Ilha A, Lardosa E, Figueiredo P, Oliveira RF. A Reserva da Biosfera da Mata Atlântica no Estado do Rio de Janeiro. $2^{\text {rd }}$ ed. Rio de Janeiro: Conselho Nacional da Reserva da Biosfera da Mata Atlântica, 2003. 61 p.

Rio de Janeiro (Estado). Tribunal de Contas do Estado do Rio de Janeiro - TCE-RJ. Secretaria Geral do Planejamento, Tribunal de Contas do Estado do Rio de
Janeiro. Estudo sócio-econômico 2004, Itaguaí. Rio de Janeiro; 2004. 164 p.

Rocha CFD, Bergallo HG, Alves MAS, van Sluys M. A biodiversidade nos grandes remanescentes florestais do estado do Rio de Janeiro e nas restingas da Mata Atlântica. São Carlos: RiMa; 2003. 160 p.

Silva GC, Nascimento MT. Fitossociologia de um remanescente de mata sobre tabuleiros no norte do estado do Rio de Janeiro (Mata do Carvão). Revista Brasileira de Botânica 2001; 24(1)51-62.

Souza VC, Lorenzi H. Botânica Sistemática: guia ilustrado para identificação das famílias de Angiospermas da flora brasileira, baseado em APGII. $2^{\text {rd }}$ ed. Nova Odessa: Instituto Plantarum; 2005. 640 p.

Souza GR. Florística do estrato arbustivo arbóreo em um trecho de floresta atlântica no médio Paraíba do Sul, município de Volta Redonda, Rio de Janeiro [dissertação]. Seropédica, RJ: Instituto de Florestas, Universidade Federal Rural do Rio de Janeiro; 2002.

Valcarcel R, D’altério CF. Medidas físico-biológicas de recuperação de áreas degradadas: avaliação das modificações edáficas e fitossociológicas. Floresta $e$ Ambiente 1998; 5(1)68-88.

Veloso HP, Rangel Filho ALR, Lima JCA. Classificação da vegetação brasileira, adaptada a um sistema universal. Manual Técnico da Vegetação brasileira. Rio de Janeiro: Departamento de Recursos Naturais e Estudos Ambientais, Fundação Instituto Brasileiro de geografia e Estatística; 1991. 124 p.

Vuono YS. Inventário Fitossociológico. In: Sylvestre LS, Rosa MMT, organizadores. Manual metodológico para estudos botânicos na Mata Atlântica. Seropédica: Editora Universidade Rural; 2002. p. 51-65. 